\title{
Arbor
}

\section{La calidad en el entorno hospitalario. Calidad e incentivos}

\section{Vicente Gil Suay}

Arbor CLXX, 670 (Octubre 2001), 339-352 pp.

Tras el planteamiento del modelo europeo de calidad como una vía sólida para conseguir mejorar el desarrollo de la misión de los hospitales, se profundiza sobre la situación actual y un futuro con la necesaria implicación de todos los actores del sistema, financiadores, proveedores, profesionales y ciudadanos.

\section{Introducción}

Es sabido que las profesiones sanitarias han mostrado a lo largo de su existencia una especial preocupación por la calidad de su trabajo y prueba de ello es el avance tan significativo que han experimentado las ciencias de la salud, sobretodo en las últimas décadas, liderando el mundo de las publicaciones científicas. Pero no es menos cierto que este desarrollo tan espectacular se ha producido en lo que se conoce como ámbito cientifico-técnico. Sin embargo no ha sido tanta la mejora producida en los aspectos que rodean a la traslación de esos avances a nuestros ciudadanos. Nos referimos, especialmente, a la percepción que por parte de ellos se produce de que la asistencia se ha deshumanizado. Superada con creces la etapa en que la medicina tenía que demostrar su capacidad de curar a los pacientes (asistimos ahora a una etapa en la que la sociedad espera que TODO se cure) 
los ciudadanos reclaman, legítimamente, un mejor trato, más información y participación en su proceso, mayor celeridad en la resolución de sus problemas, mayor confort, etc. ya que la posibilidad de curación se da por supuesta.

Es en este último aspecto, que podemos ligar a la calidad percibida, en el que debemos realizar un gran esfuerzo para estar a la altura de lo que nuestros clientes demandan y que permitirá a nuestras organizaciones mantenerse en el tiempo, en un entorno que cada vez se nos presenta más agresivo.

Precisamente, la necesidad de adaptarse a las nuevas exigencias de calidad en la prestación de la asistencia a nuestros ciudadanos es lo que nos ha llevado a implantar la cultura de la calidad, no sólo científico-técnica, en nuestras organizaciones.

Al igual que en otros sectores, pero quizás con evidente retraso respecto a ellos, el concepto y aplicación de la calidad en nuestros hospitales ha pasado por sucesivas etapas. Primero fue el Control de la Calidad, centrado en revisar si lo que realizábamos, en términos de resultado, era lo adecuado y esperado. Después, y ante la escasez de respuesta a la demanda obtenida con la introducción del Control de Calidad, se introduce el Aseguramiento de la Calidad, centrado en analizar si lo que realizamos lo hacemos como corresponde al estándar o norma establecida. Finalmente, se ha dado un paso definitivo al introducir la Calidad Total en nuestros centros. La Calidad Total abarca todos los aspectos de la organización y revisa desde el liderazgo y la estrategia, pasando por los procesos, a los resultados frente a los empleados, los clientes, la sociedad y, por supuesto, financieros. La Calidad Total nace a partir de los años 50 en Japón, de la mano de los americanos Deming y Juran, discípulos de Shewhart, que desencadenan un amplio movimiento al que se incorporan otros prestigiosos autores como Ishikawa, Taguchi, Ohno, etc. El concepto y aplicación de la Calidad Total podemos decir que engloba al Aseguramiento y al Control de la Calidad en un todo que se extiende por toda la organización.

En las páginas siguientes vamos a recorrer lo que ha sido la aplicación de estos conceptos en nuestros hospitales y lo que puede aportar la calidad en la motivación de nuestros profesionales.

\section{El control de la calidad}

Definido el Control de la Calidad como el conjunto de técnicas y actividades, de carácter operativo, utilizadas para verificar los requisitos 
relativos a la calidad del producto o servicio, conviene recordar cómo se ha venido aplicando este concepto en nuestros hospitales.

Esta etapa se desarrolló en nuestros centros, fundamentalmente, a lo largo de los años 80 y principios de los 90 y supuso para muchos de ellos, o al menos para los considerados como grandes hospitales, la creación de las llamadas «Unidades de Control de la Calidad».

$\mathrm{El}$ papel de estas unidades fue variando en el tiempo. Al principio centraron su actuación en la recogida y evaluación de los principales indicadores de actividad del centro, elaborando informes apropiados para la gestión del hospital. Así se recogían los ingresos, estancias, estancia media, consultas, intervenciones, etc. y se analizaban las tendencias de dichos indicadores. Posteriormente, a estos indicadores, se fueron añadiendo otros cuya finalidad era la de servir de alarma o reflexión sobre el nivel de calidad con el que la organización realizaba la actividad, añadiéndose indicadores como el porcentaje de fallecimientos, los reingresos, el porcentaje de necropsias, las úlceras por decúbito o más recientemente las tasas de infección nosocomial.

Como se puede apreciar, el énfasis se pone en el control del producto final, tal como había ocurrido en otros sectores de la producción en décadas anteriores, y la principal herramienta utilizada era la estadística. No obstante, con los últimos indicadores referidos, comienza a aparecer la necesidad de revisar la práctica clínica para comprobar si se ajusta o no a normas preestablecidas. Aparece en nuestro sistema sanitario la necesidad de revisar los procesos en lugar de revisar o controlar los productos finales y con ello la necesidad de «asegurar o acreditar» que se realizan con la máxima calidad.

Paralelamente a esta evolución se incorporan, dentro de las Unidades de Control de la Calidad, las llamadas Comisiones de Garantía de la Calidad. Estas comisiones nacen, fundamentalmente, de la necesidad percibida de crear grupos de expertos dentro del hospital que asesoren a la Dirección sobre las políticas a desarrollar en cada una de las áreas en las que se constituyen. Así nacen las comisiones de Tumores, Infecciones y Política Antibiótica, de Mortalidad, Farmacia, etc. La herramienta utilizada es, con frecuencia, la elaboración de protocolos y se podría decir que representan el primer esbozo de los grupos de mejora de un modelo de calidad total. Si bien es cierto que con la elaboración de los protocolos se ha mejorado en la limitación de la variabilidad de la práctica clínica, no es menos cierto que la orientación de los mismos ha sido, casi exclusivamente, hacia la calidad científico-técnica, relegando a un segundo plano otros aspectos de la calidad de gran importancia para los ciudadanos, para los financiadores del 
sistema o para los propios empleados. Éstas son, entre otras, las razones por las que el modelo de Control de la Calidad resulta insuficiente para conseguir la excelencia de la organización.

\section{El aseguramiento de la calidad}

Por Aseguramiento de la Calidad entendemos el conjunto de acciones, planificadas y sistemáticas, que son necesarias para proporcionar la confianza adecuada de que un producto o servicio va a satisfacer unos requisitos determinados de calidad. Para ello, es necesario que exista una «norma» o «estándar» explicitada y conocida que contenga, de forma precisa, los requisitos de calidad a cumplir, así como una agencia o ente externo a esa empresa que certifique que la norma o el estándar se ha alcanzado.

Dentro del mundo hospitalario el sistema de aseguramiento más conocido es el establecido por la Joint Commission on Acreditation of Healthcare Organitations (Joint Commission) que desde 1951 viene elaborando estándares para acreditar a aquellos hospitales que cumplen todos los requisitos contenidos en la norma. Estos estándares han evolucionado desde más estructurales al principio, a más funcionales en la actualidad, evaluando el conjunto del funcionamiento del hospital.

El estándar es la pieza básica de este sistema. Cada estándar va seguido de un «propósito» que explica el sentido de aquél. El conjunto estándar y propósito forman la base que sirve para la evaluación del hospital. Se evalúan catorce estándares agrupados en tres funciones:

a) Función centrada en el paciente, con los estándares siguientes: Derechos del paciente, evaluación del paciente, asistencia al paciente, educación del paciente y su familia y continuidad de la asistencia.

b) Funciones de la organización, con los estándares: Mejoras en los resultados de salud de los pacientes, liderazgo, gestión del entorno de la asistencia, gestión de los recursos humanos, gestión de la información, y vigilancia, prevención y control de la infección.

c) Funciones de la Dirección que comprende los estándares de: Órganos de gobierno, cuadro médico y enfermería.

La acreditación, formalizada mediante la correspondiente certificación, que emite la Joint Commission tiene su mayor desarrollo en EEUU, en donde se inició, como se ha dicho, en los años cincuenta. También algunos hospitales europeos han optado realizar sus procesos de mejora basándose en esta metodología de trabajo, incluyendo España 
donde la Fundación Avedis Donabedian es la encargada de realizar la correspondiente certificación de los centros.

Otro de los procedimientos de Aseguramiento de la Calidad, muy introducido en otros sectores de la producción, y de forma creciente en el sector sanitario, es el aseguramiento mediante la Norma ISO ( International Standard Organitation). Las más conocidas son la serie 9000. $\mathrm{Al}$ igual que el estándar, la norma proporciona una descripción de cómo una actividad o conjunto de actividades han de llevarse a cabo de modo que pueda obtenerse la excelencia en el resultado. La internacionalización de las normas o reglas refuerza y garantiza la confianza de los clientes y de la propia organización que las cumple. Las más conocidas son la 9001 y 9002. La 9001 recoge veinte apartados diferentes a revisar y que van desde la responsabilidad de la dirección en establecer políticas de calidad hasta el servicio posventa, pasando por un exhaustivo análisis de los procesos. La ISO 9002 es otra de las normas más utilizadas y sobre todo en el sector servicios. Destaca el papel de la dirección en el liderazgo y compromiso con la calidad y la orientación hacia la búsqueda de la satisfacción del cliente. Los recursos humanos, su motivación y adecuación son, junto a los recursos materiales, otro de los aspectos destacables. La interacción y en especial la comunicación entre los clientes, tanto externos como internos, cobra especial importancia.

En el H. U. La Fé se están desarrollando en la actualidad los trabajos necesarios para obtener la certificación por la Norma 9002 en tres áreas, la Unidad de Coagulopatías Congénitas, el Comité de Ensayos Clínicos y el proceso de suministro desde los almacenes generales.

No cabe duda que el propósito del Aseguramiento de la Calidad, como se puede observar, es doble. Por una parte, el aseguramiento interno con el que se trata de dar confianza a la dirección de la organización. De otra, el aseguramiento externo que pretende proporcionar confianza y satisfacción al cliente externo. El núcleo central del aseguramiento lo constituye la garantía de la calidad en todas y cada una de las fases del desarrollo del proceso.

\section{La calidad total. El modelo europeo de calidad total.}

Ya se comentó en la introducción que la evolución de la calidad ha pasado por el Control de la Calidad, centrado en comprobar los requisitos que un producto debía cumplir al final del proceso de fabricación y elaboración; aparece después el Aseguramiento de la Calidad que va más allá al asegurar que los procesos, y no sólo el resultado, se desarrollan de acuerdo con la norma; y finalmente, se introduce 
el concepto de Calidad Total en el que la organización se propone satisfacer todas las necesidades y expectativas de sus clientes, sus empleados, las entidades financiadoras y la sociedad en general.

La introducción de la Calidad Total en las organizaciones se basa en los llamados «Principios de la Calidad Total»:

a) Liderazgo: La Dirección de debe asumir el liderazgo en la gestión de la calidad y desplegarla en toda la empresa, de forma que se logre integrar la calidad en la cultura de toda la organización. La Dirección transmite esta cultura de la calidad y facilita los medios para su implantación: Comunicación, formación, recursos, etc.

b) Orientación al cliente: La organización busca la satisfacción del cliente ganando su confianza y fidelidad, además de procurar adaptarse a los cambios en las necesidades de aquellos ya que la satisfacción no es estática. c) Participación de todas las personas: Todos los empleados realizan actividades diarias que aportarán valor al cliente. Obtener la participación de todos y cada uno de los empleados es vital para que el proceso de mejora pueda llevarse a cabo y el cliente lo perciba. También los propios empleados se transforman en clientes internos al ser proveedores y receptores de procesos. Además, el trabajo en equipo es fundamental, propiciando las sinergias y las relaciones interdepartamentales.

d) Aplicación de la mejora continua a todas las actividades: El concepto de mejora continua nace con Shewhart al describir su conocido ciclo PDCA (Plan-Do-Check-Act), extendido posteriormente por Deming: Planificar, identificando los procesos y analizando las áreas de mejora, estableciendo objetivos e indicadores a controlar. Hacer, es decir, realizar lo que se ha planificado. Comprobar, analizando si los resultados obtenidos responden a los objetivos marcados. Actuar o ajustar, corrigiendo las desviaciones producidas en los resultados e iniciando un nuevo ciclo hacia una nueva mejora del proceso (fig. 1)

Figura 1

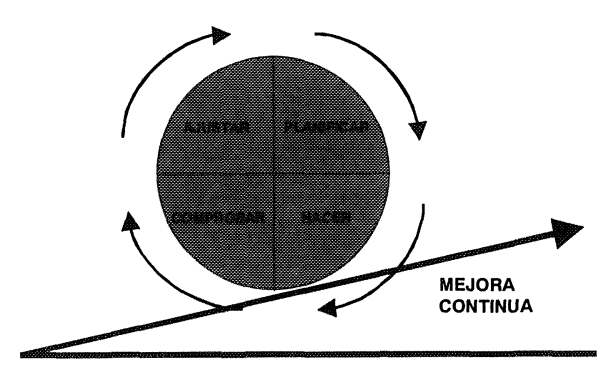


e) Gestión de la actividad en términos de proceso: En lugar de gestionar la organización por funciones o departamentos, se gestiona por los diferentes procesos que se desarrollan en una organización, identificándolos, reorganizándolos, revisándolos y proponiendo las mejoras necesarias.

f) Gestión mediante indicadores: Se evita la subjetividad de las opiniones y se sustituye por datos objetivos que permitan realizar la toma de decisiones.

Estos principios se desarrollan en diferentes modelos de Gestión de la Calidad Total de los que podríamos señalar, entre los más conocidos, el Modelo Deming (1951) implantado en Japón, el Modelo Malcom Balridge (1987), en los EEUU, y en Europa el Modelo Europeo de Gestión de la Calidad Total (1988), creado por la Fundación Europea para la Gestión de la Calidad, más conocido por las siglas E.F.Q.M. $\mathrm{y}$ al que nos vamos a referir ahora.

El modelo promueve la autoevaluación de la organización siguiendo un análisis exhaustivo y sistemático de todas sus actividades. Si analizamos el esquema que la asistencia sanitaria ha venido siguiendo últimamente en la gestión de la calidad vemos que se ha basado en el propuesto por Donabedian y que se compone del análisis de la calidad de la estructura, del proceso y del resultado. Este esquema no difiere mucho del propuesto por el modelo EFQM (fig. 2) que se puede decir que contiene dos núcleos fundamentales: Los capacitadores o agentes que incluye la revisión de todos los medios que se emplean para conseguir los resultados de la organización, y los resultados que abarca el análisis de los que se debe conseguir y se está consiguiendo.

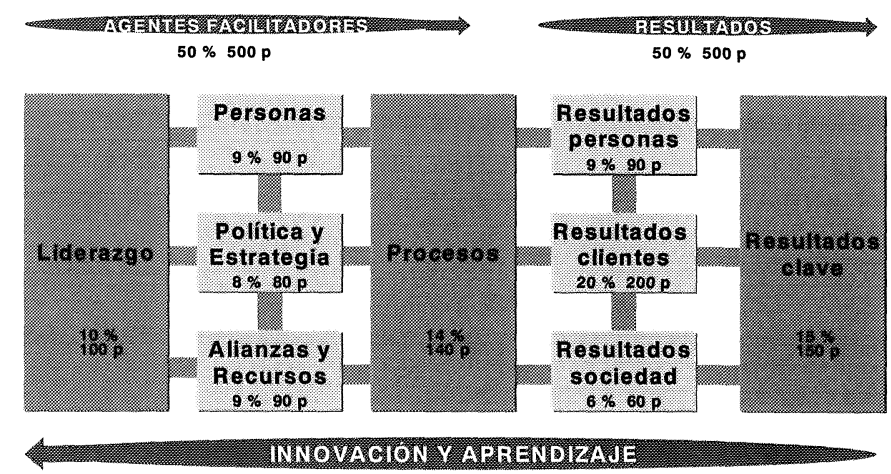


La revisión de los agentes del modelo incluye la revisión de los cinco criterios que lo componen:

- Criterio 1: Liderazgo. Analiza cómo los líderes desarrollan y facilitan la consecución de la misión y la visión, desarrollan los valores necesarios para alcanzar el éxito a largo plazo e implantan todo ello en la organización mediante las acciones y comportamientos adecuados, estando implicados personalmente en asegurar que el sistema de gestión de la organización se desarrolla e implanta.

- Criterio 2: Política y Estrategia. Cómo implanta la organización su misión y visión mediante una estrategia claramente centrada en todos los grupos de interés y apoyada por políticas, planes, objetivos, metas y procesos relevantes.

- Criterio 3: Personas. Cómo gestiona, desarrolla y aprovecha la organización el conocimiento y todo el potencial de las personas que la componen, tanto a nivel individual como de equipos o de la organización en su conjunto; y cómo planifica estas actividades en apoyo de su política y estrategia y del eficaz funcionamiento de sus procesos.

- Criterio 4: Alianzas y Recursos. Cómo planifica y gestiona la organización sus alianzas externas y sus recursos internos en apoyo de su política y estrategia y del eficaz funcionamiento de sus procesos.

- Criterio 5: Procesos. Cómo diseña, gestiona y mejora la organización sus procesos para apoyar su política y estrategia y para satisfacer plenamente, generando cada vez mayor valor, a sus clientes y otros grupos de interés.

Los resultados se valoran mediante el análisis de los cuatro criterios siguientes:

- Criterio 6: Resultados en los clientes. Analiza qué logros esta alcanzando la organización en relación con sus clientes externos.

- Criterio 7: Resultados en las personas. Qué logros está alcanzando la organización en relación con las personas que la integran.

- Criterio 8: Resultados en la sociedad. Qué logros esta alcanzando la organización en la sociedad, en el ámbito local, nacional o internacional (según resulte pertinente).

- Criterio 9: Resultados clave. Qué logros esta alcanzando la organización con relación al rendimiento planificado.

Cada uno de los criterios se divide a su vez en subcriterios que deben tenerse en cuenta a la hora del análisis y que permite evaluar la totalidad del concepto expresado en el criterio. La evaluación del subcriterio, en el caso de los agentes, consiste en el análisis del enfoque, despliegue, y evaluación y revisión del enfoque y su despliegue; en el caso de los resultados, en cada subcriterio se analiza las tendencias, 
el logro de los objetivos, la comparación de los resultados con otras organizaciones (benchmarking), y si los resultados son consecuencia del enfoque y abarcan las áreas relevantes. Cada una de estos aspectos son puntuados, mediante una metodología rigurosa, en función de su consecución, obteniéndose para cada criterio una puntuación (la máxima es la contenida en cada recuadro) y con la suma de todos los criterios se obtiene la puntuación final de la autoevaluación. Esta puntuación es útil, no sólo porque proporciona un elemento de comparación entre las organizaciones sino, también, porque permite a la propia organización analizar su evolución en la mejora continua a lo largo del tiempo. Obviamente, las puntuaciones deben ser ratificadas por un equipo externo experto en la evaluación del modelo. Anualmente, la Fundación Europea para la Gestión de la Calidad convoca unos premios de calidad para estimular a las diferentes organizaciones a la implantación del modelo y con ello contribuir al desarrollo de la Calidad Total. Hay que resaltar la «dureza» del modelo ya que las organizaciones que alcanzan estos premios y son consideradas excelentes se sitúan entre los 600 y 700 puntos sobre los 1000 potenciales. Puntuaciones de 300 a 400 puntos son consideradas aceptables siendo laboriosa la progresión a partir de dichas cifras.

Figura 3

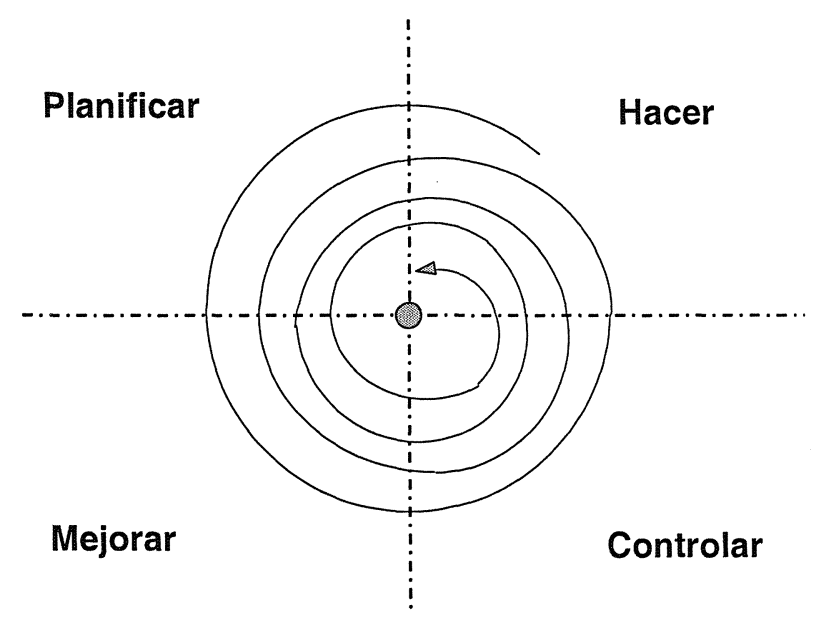

Pero si es importante conocer la puntuación final, más importante resulta poder extraer del análisis los puntos débiles de la organización, lo que permite establecer diferentes planes de mejora a poner en marcha por todos los integrantes de la misma (modelo basado en la participación y trabajo en equipo). Estos planes de mejora deben priorizarse mediante la correspondiente matriz de priorización y desplegarse en todos los ámbitos de la organización. Cuando la implantación de los planes de 
mejora lo aconsejen se podrá realizar una nueva autoevaluación que nos informará de los avances conseguidos y al mismo tiempo nos descubrirá nuevas áreas de mejora, entrando en una espiral de mejora continua de la calidad (fig. 3).

La aplicación de este modelo permite pasar de una Gestión Basada en la Dirección Por Objetivos a una Gestión Basada en la Calidad Total. La finalidad ya no es tanto la consecución de los objetivos sino que éstos se obtienen como consecuencia de la aplicación de una nueva filosofía basada en lograr la satisfacción de los clientes, de las personas de la propia organización, de los financiadores y de la sociedad en general con el mínimo coste.

En la actualidad son muchos los centros sanitarios de nuestro país que están aplicando este modelo de calidad total y para el lector interesado aconsejamos la lectura del número monográfico que la revista Calidad Asistencial dedicó a este tema (Rev. Calidad Asistencial, Abril 2000; 15: 129-191).

\section{Calidad, motivación e incentivos}

El conocimiento del modelo europeo de calidad nos permite adentrarnos por los mecanismos con los que la organización favorece a sus empleados, motivándolos y consiguiendo su alianza en la consecución de los objetivos. Una de las limitaciones principales que tiene el Control de la Calidad o el sistema de aseguramiento de la misma (aunque en menor grado) o, incluso, la Dirección Por Objetivos es la escasa atención que dedican a la motivación e implicación del personal. Centran su atención, según el caso, en el resultado final del producto, en la calidad del proceso o, en el último caso, en el logro de los objetivos, sobretodo a corto plazo. Por el contrario, el modelo europeo dedica una importancia capital a este tema que aborda en el criterio1: Liderazgo, el 2: Política y estrategia, el 3: Personas y el 7: Resultados en las personas.

Así, en el criterio 1 el subcriterio 1d revisa la motivación, apoyo y reconocimiento de las personas de la organización por parte de los líderes de la misma. Valora hasta que punto los líderes se preocupan por comunicar la misión, visión, valores, política y estrategia, planes, objetivos y metas de la organización a las personas que la integran; si los líderes son accesibles y escuchan activamente y responden a las personas; si ayudan y apoyan a las personas a conseguir sus planes, objetivos y metas; si permiten y animan a la participación activa y 
si reconocen, oportuna y adecuadamente, los esfuerzos de individuos $\mathrm{y}$ equipos.

El criterio 2 que analiza la política y estrategia de la organización, dedica el subcriterio 2 a a revisar, entre otras actividades, si la organización comprende las necesidades y expectativas de los empleados y las incorpora en la estrategia de la propia organización.

$\mathrm{El}$ criterio 3 se dedica por entero a revisar el factor recursos humanos de la organización. A lo largo de sus cinco subcriterios analiza si se planifican, gestionan y mejoran esos recursos buscando la implicación de las personas y sus representantes; si se selecciona de forma justa e imparcial a los mejores para incorporarse a los nuevos puestos; si se mejoran las estructuras organizativas dirigiéndolas hacia modelos más matriciales; si se desarrollan y utilizan planes de formación y desarrollo de habilidades; si se estimula el trabajo en equipo; si se evalúa el rendimiento de las personas y se ayuda a mejorarlo; si se fomentan los comportamientos creativos e innovadores; si se faculta a las personas de la organización a emprender acciones y se valora su eficacia; si se fomenta la comunicación ascendente y descendente y el diálogo; si existen recompensas, reconocimientos y se establecen beneficios sociales, como planes de pensiones, seguros, etc.

El criterio 7 analiza la satisfacción que obtienen las personas de la organización como consecuencia del despliegue de las políticas contenidas en los criterios antes mencionados. En este punto es imprescindible el uso de herramientas como las encuestas, entrevistas personales o evaluaciones y mediante ellas conocer el grado de motivación y satisfacción de los empleados respecto de temas tan cruciales como el desarrollo de la carrera profesional, la asunción y delegación de responsabilidades, la igualdad de oportunidades, el reconocimiento, las condiciones de empleo, de seguridad e higiene en el trabajo, de seguridad en el puesto, de salario y beneficios, etc.

Sin duda que la aplicación de estas políticas en el entorno de los servicios sanitarios producirá un incremento de la motivación y satisfacción de quienes trabajamos en él al conseguir una mayor autoestima y nivel de realización personal que son, precisamente, el vértice de la conocida pirámide de necesidades de Maslow (fig. 4).

En cuanto a los incentivos que el propio sistema establece para conseguir los objetivos podríamos decir que son más propios de la DPO que de una Gestión de la Calidad Total sin que ello suponga que la incentivación no tenga cabida en dicho modelo, pero estaríamos de acuerdo con Deming cuando señala que es poco eficiente incentivar a los trabajadores a conseguir aquello que, al depender de fases del 


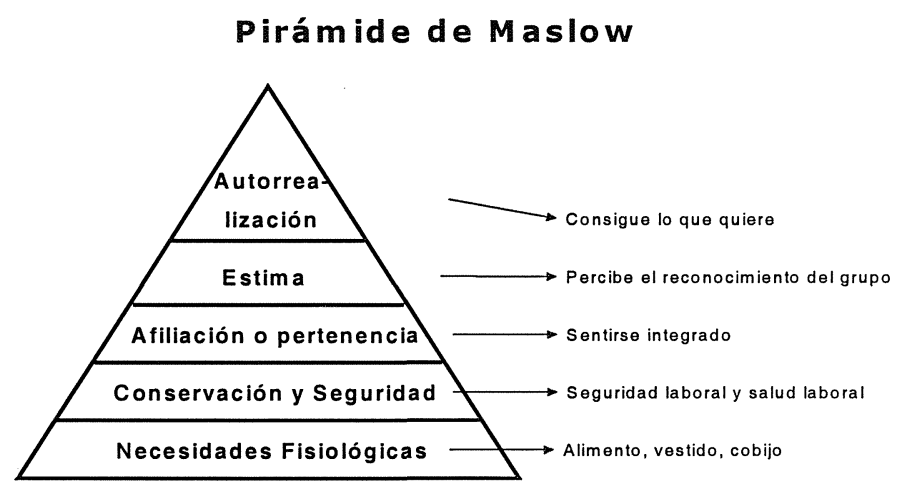

proceso que no están bajo su control, difícilmente podrán conseguir. La incentivación debería dirigirse hacia la participación de los empleados en los diferentes planes de mejora que se obtienen al aplicar el modelo europeo en la organización.

En nuestro sistema sanitario existen no pocas limitaciones para llevar a cabo una gestión adecuada de los recursos humanos. Ejemplo de ello lo tenemos en el acceso al puesto de trabajo, mediante el concurso-oposición y con carácter vitalicio; el régimen estatutario y rígido que regula las relaciones de la empresa con los empleados y que se acompaña de un régimen retributivo no diferencial; diferentes retribuciones para algunos territorios autonómicos; incentivación por parte de otros agentes del sistema, especialmente industria farmacéutica y de equipamiento médico, lo que puede facilitar la introducción y uso de tecnología sanitaria que no tiene por qué coincidir con las necesidades e intereses del sistema, etc.

Frente a esta situación se han venido implantando algunas soluciones para que, aún dentro de un marco jurídico-administrativo tan rígido, se pueda lograr la introducción de acciones motivadoras-incentivadoras. En este sentido cabe señalar ejemplos como:

- La introducción del llamado «Contrato-Programa» que contempla, entre otros aspectos, la posibilidad de establecer una retribución variable en función de la productividad y consecución de objetivos y también, en algunos casos, la posibilidad de invertir los «ahorros» en la gestión del presupuesto en la adquisición de equipamiento, formación, etc.

- Introducción de un sistema de retribución diferente en las Fundaciones y Empresas Públicas Sanitarias. En estas instituciones, debido a que 
gozan de personalidad jurídica propia, la relación contractual se basa en el régimen laboral y permite establecer un sistema retributivo que reconoce una parte fija en función del grupo profesional al que se pertenece, un complemento de puesto de trabajo, diferente según la categoría de facultativo o personal sanitario o administrativo, etc., un complemento personal, en función de la realización de labores de gestión, guardias, etc. y un incentivo variable que se retribuirá en función de la consecución de una serie de objetivos de actividad, calidad y eficiencia.

- Desarrollo de la carrera profesional. Existen ejemplos en nuestro medio de propuestas de cambios en la carrera profesional en los que se sustituye el marco actual de Jefe de Servicio, Sección y Facultativo Especialista por una mayor gradación, estableciendo un mayor número de niveles, generalmente del I al V, y con diferentes requisitos de acceso y también de retribuciones ligadas a cada uno de ellos. Ejemplos de ellos los podemos encontrar en La Comunidad Foral de Navarra, el Hospital Gregorio Marañón de Madrid o el Hospital Clínico de Barcelona. - Introducción de la Gestión Clínica, como cambio del modelo organizativo asistencial, pasando a estructuras más horizontales (matriciales) que sustituyan a la ya caduca verticalidad de la unidad asistencial. Este cambio organizativo permite transferir a la unidad mayor autonomía y capacidad de decisión, establecer una carrera profesional diferente, establecer objetivos de actividad y calidad específicos por áreas que además pueden servir de base para un sistema retributivo diferente.

En cualquier caso se hace necesario establecer una reflexión profunda sobre la situación actual y el futuro que deseamos y para ello será necesario recoger la opinión de todos los actores del sistema, financiadores, proveedores, profesionales y clientes con el objeto de conseguir la necesaria implicación de todos. El modelo europeo de calidad aparece a primera vista, y a juzgar por las experiencias desarrolladas, una vía sólida para conseguirlo.

\section{Bibliografía}

1 MaIz, E.. Gestión de la Calidad. Diploma en Gestión de Servicios Clínicos. Valencia. 1997.

2 De La Quintana, F. Programas de calidad en los hospitales españoles: Perspectivas de los profesionales de la salud. 1997.

3 Joint Commission. Estándares de Acreditación de Hospitales. Fundaçio Avedis Donabedian. 1997.

4 European Foundation for Quality Management. Modelo EFQM de Excelencia. Club de Gestión de la Calidad. 1999.

5 MuÑoz, A. La gestión de la calidad total en la Administración Pública. Editorial Diaz de Santos. 1999. 


\section{Vicente Gil Suay}

352

6 Modelo Europeo de Excelencia (EFQM) Rev. Española de Calidad Asistencial. Abril 2000; 15: 129-191.

7 MuÑoz A.. Dirección por objetivos y gestión de la calidad total. Todo Hospital. $1996 ; 132 ; 43-49$.

8 Ibern, P. Incentivos y contratos en los servicios de salud. Springer-Verlag. Barcelona.1999.

9 GARCIA, B. Algunos apuntes sobre incentivos en los hospitales públicos. Todo Hospital. 1997; 141: 13-21.

10 CARRetero L.. Herramientas para la gestión clínica. Gestión Hospitalaria. 2000; 11 (1): 15-21. 